\title{
BMJ Open Convergent parallel mixed-methods study to understand information exchange in paediatric critical care and inform the development of safety- enhancing interventions: a protocol study
}

\author{
Jessica Tomasi, ${ }^{1}$ Carly Warren, ${ }^{1,2}$ Lauren Kolodzey, ${ }^{1}$ Sonia Pinkney, ${ }^{3}$ \\ Anne-Marie Guerguerian, ${ }^{4}$ Roxanne Kirsch, ${ }^{4}$ Jackie Hubbert, ${ }^{4}$ Christina Sperling, ${ }^{4}$ \\ Patricia Sutton, ${ }^{4}$ Peter Laussen, ${ }^{4}$ Patricia Trbovich ${ }^{1,2,3}$
}

To cite: Tomasi J, Warren C Kolodzey L, et al. Convergent parallel mixed-methods study to understand information exchange in paediatric critical care and inform the development of safetyenhancing interventions: a protocol study. BMJ Open 2018;8:e23691. doi:10.1136/ bmjopen-2018-023691

- Prepublication history and additional material for this paper are available online. To view please visit the journal (http:// dx.doi.org/10.1136/bmjopen2018-023691).

Received 18 April 2018 Revised 22 June 2018 Accepted 8 August 2018

D) Check for updates

(C) Author(s) (or their employer(s)) 2018. Re-use permitted under CC BY-NC. No commercial re-use. See rights and permissions. Published by BMJ.

For numbered affiliations see end of article.

Correspondence to Dr Patricia Trbovich; patricia.trbovich@utoronto.ca

\begin{abstract}
Introduction The effective exchange of clinical information is essential to high-quality patient care, especially in the critical care unit (CCU) where communication failures can have profoundly negative impacts on critically ill patients with limited physiological capacity to tolerate errors. A comprehensive systematic characterisation of information exchange within a CCU is needed to inform the development and implementation of effective, contextually appropriate interventions. The objective of this study is to characterise when, where and how healthcare providers exchange clinical information in the Department of Critical Care Medicine at The Hospital for Sick Children and explore the factors that currently facilitate or counter established best rounding practices therein.
\end{abstract}

Methods and analysis A convergent parallel mixedmethods study design will be used to collect, analyse and interpret quantitative and qualitative data. Naturalistic observations of rounds and relevant peripheral information exchange activities will be conducted to collect time-stamped event data on workflow and communication patterns (time-motion data) and field notes. To complement observational data, the subjective perspectives of healthcare providers and patient families will be gathered through surveys and interviews. Departmental metrics will be collected to further contextualise the environment. Time-motion data will be analysed quantitatively; patterns in field note, survey and interview results will be examined based on themes identified deductively from literature and/or inductively based on the data collected (thematic analysis). The proactive triangulation of these systemic, procedural and contextual data will inform the design and implementation of efficacious interventions in future work.

Ethics and dissemination Institutional research ethics approval has been acquired (REB \#1000059173). Results will be published in peer-reviewed journals and presented at relevant conferences. Findings will be presented to stakeholders including interdisciplinary staff, departmental
Strengths and limitations of this study

- This protocol takes a human factors research approach to understand complex interactions of work system elements that influence the exchange of clinical information.

- The mixed-methods study design captures objective and subjective data around current communication processes from a wide variety of interdisciplinary and family stakeholders and focuses on existing effective practices as well as opportunities for improvement.

- Knowledge of the current clinical work system will be cultivated proactively to generate evidence for the development and implementation of effective and appropriate interventions.

- As participation in this study is voluntary, a selection bias may exist, and since data collection is limited to one department within one institution, results may not be generalisable.

- It is possible that some communication events may not be captured, due to the fast-paced and complex critical care unit environment.

management and leadership and families to highlight the strengths and weaknesses of the exchange of clinical information in its current state and develop user-centred recommendations for improvement.

\section{INTRODUCTION}

Communication errors are one of the most common causes of adverse medical events, resulting in various impacts including adverse drug reactions, hospital-acquired infections, sepsis and unplanned returns to the operating theatre. ${ }^{1-4}$ Communication failures can have an especially negative impact on 
critically ill patients with limited physiological capacity to tolerate errors. ${ }^{56}$ In the critical care unit (CCU), the delivery of safe, high-quality patient care relies on strong collaboration between the interdisciplinary care team. ${ }^{578}$ The effective exchange of clinical information, through structured daily rounds and handover, is essential to providing safe and effective care. ${ }^{8-10}$

Lane $e t a t^{6}$ conducted a systematic review of evidence-informed practices for patient care rounds in the CCU. They identified 13 facilitators of and 9 barriers to conducting high-quality rounds; the literature presented strong evidence for implementing streamlined multidisciplinary rounds using a checklist, in a standard location and at a standard time, with explicit roles defined for each participating healthcare provider (HCP). Based on their results, Lane et al compiled an itemised list of recommended best practices to improve rounds, ranked by strength of recommendation. In 2015, Holodinsky et a $\ell^{\ominus}$ conducted a cross-sectional survey of Canadian adult medical/surgical CCUs with purposefully sampled follow-up interviews to describe the structure, process and outcomes of rounds and explore whether Lane $e t$ al s best practices are used in daily patient care. They discovered differential adoption of recommended practices and identified the following areas for improvement in addition to those listed by Lane et al: identifying and ensuring the engagement of essential participants, determining the role of patients and families in rounds, modifying the role of teaching and developing a metric for measuring rounding quality. Tripathi $e t a l^{11}$ incorporated the strategies proposed by Lane et al to promote open, honest and unbiased communication between patients, their families and all HCPs and to enhance patient-centred care in their paediatric CCU. Although several practical and low-risk 'best practices' for CCU rounds have been identified based on existing literature ${ }^{6}$ and implemented variably since their publication, ${ }^{911}$ little work has been done to understand the department-specific factors that may facilitate, hinder or contraindicate their implementation.

The overarching goals of the proposed study are to characterise the exchange of clinical information in the Department of Critical Care Medicine at The Hospital for Sick Children (SickKids) and to explore the objective and perceived systemic facilitators and barriers to efficacy thereof. In doing so, we will determine the extent to which established 'best practices' are currently implemented for rounds, assess their appropriateness based on departmental context, including consideration of peripheral information exchange activities, and collect evidence to inform the future design and implementation of user-centred recommendations for improvement.

\section{The complexity of clinical information exchange}

Given the established significance of effective communication and collaboration with respect to patient care and outcomes, much research has been dedicated to studying the structured instances of information exchange that are used to transfer clinical data between HCPs.
'Rounds' is an all-encompassing term that usually includes any regularly scheduled meeting during which clinical problems encountered are discussed. ${ }^{12} 13$ Rounds can be classified by content, location or attendees (eg, bedside rounds) ${ }^{14}$ while teaching rounds (eg, grand rounds) usually take place off the ward and are delivered in a lecture format. ${ }^{15}$ Interdisciplinary rounds involve all members of the care team and provide an opportunity to discuss and coordinate care between physicians, nurses, pharmacists and others. ${ }^{16}$ Handover is a within-discipline process that can be defined as 'the real-time process of passing patient-specific information from one HCP to another at the end of a working shift'. ${ }^{17}$

The main content that is exchanged during interdisciplinary rounds and handover is patient-specific information. Often this information is organised by body system; updates will be given on a patient's neurological condition, respiratory condition, renal function and so on. ${ }^{18}$ Patient-specific information may also include information such as case history, treatment plan and discharge plan. ${ }^{18}$ In addition to the exchange of this information, rounds may be used as an opportunity for medical education, updates to and from the patient or family and general discussion. ${ }^{18}$ The amount of rounding time that is allocated for each of these purposes is contentious, ${ }^{1319-21}$ and the order in which they are presented can vary, thus there are many potential formats for exchanging information.

The structure and process of clinical information exchange has been shown to have an impact on patient outcomes such as the incidence of preventable adverse events and mortality, as well as on other factors related to the quality of patient care including unit workflow and patient, family and provider satisfaction. ${ }^{22-25}$ A frequently reported communication error is the omission of important handover content such as medication changes or pending tests ${ }^{2627}$; however, failures of communication may be much more nuanced and complex, resulting from social factors such as the hierarchical differences of providers, inconsistent attendance and participation of rounding group, ambiguity in roles and interpersonal conflict. ${ }^{4}$ Persistent environmental distractions and interruptions, variable adherence to established best practices involving standardisation and tool use and poor information retrieval and documentation practices precipitated by suboptimal electronic medical record design are other factors cited as having the potential to negatively influence the productivity of information exchange tasks. ${ }^{6}{ }^{28}{ }^{29}$ High-quality information exchange and patient care are thus predicated on the appropriate interaction of many system elements.

Contextual factors that have contributed to department-specific rounding practices may shed light on more appropriate process changes moving forward. Research aimed at understanding the unique needs and challenges of different critical care work systems with the intention of tailoring interventions that facilitate the communication processes therein is needed to support safe, consistent and high-quality patient care. 


\section{Studying information exchange using a human factors (HF)} approach

The complex, multifaceted nature of clinical information exchange lends itself to study from a systems perspective. HF is a scientific discipline concerned with exposing and correcting elements of mismatch between humans, tools they work with and environments they work in. ${ }^{30}$

Among the most widely used models for framing the design and analysis of HF research is the Systems Engineering Initiative for Patient Safety (SEIPS) work system model. The latest iteration ${ }^{31}$ depicts the healthcare work system as a sociotechnical, human-centred system with six interacting components that together influence overall system performance:

- Person(s) (eg, individual or team characteristics, patients or staff).

- Tools and technology (eg, infusion pumps, ventilators and electronic medical records).

- Tasks (eg, simple vs complex and routine vs unanticipated).

- Organisation (eg, work schedules, training system and roles/responsibilities).

- Internal environment (eg, physical layout, light, noise and temperature).

- External environment (eg, national workforce and regulatory laws, equipment licencing and/or recalls).

By applying this systems perspective, this study aims to holistically understand the interactions that influence the implementation and success, or lack thereof, of established best practices, and subsequently inform the optimisation of system design based on human strengths and limitations and the systemic elements at play in this department.

\section{METHODS AND ANALYSIS Objectives}

1. To characterise structured instances of information exchange with a primary focus on interdisciplinary bedside rounds (ie, morning and afternoon rounds) and a secondary focus on within-discipline handover and peripheral information exchange activities.

2. To elucidate stakeholder perceptions of the current state of information exchange.

3. To compare the current practices to those recommended by Lane $e t$ al:

a. Determine which of the best practices are currently applied, with what success and as a result of which facilitators.

b. For those which are not applied, determine what is done in lieu, what barriers exist to their implementation or what reasons there are for the lack of compliance.

c. Identify any additional effective practices and opportunities for improvement.

\section{Setting}

This study will be conducted at The Hospital for Sick Children, a paediatric academic and teaching hospital located in Toronto, Canada. Data will be collected in The Department of Critical Care Medicine, which consists of 42 beds allocated to two divisions, the paediatric intensive care unit (PICU) and cardiac critical care unit (CCCU). The department admits approximately 2200 patients per year. $^{32}$

Interdisciplinary bedside rounds are conducted twice daily (07:30 and 16:00) in each unit. Morning rounds are presented by a medical trainee (fellow or resident), while afternoon rounds are presented by the nursing staff with input from the allied health providers and summarised by a medical trainee; both rounds are supervised by an attending intensivist. Within-discipline patient handovers include nurse, charge nurse, respiratory therapist (RT) and charge RT handovers that take place twice daily (07:00 and 19:00) and evening rounds each night at 22:00. Teaching rounds away from the bedside (09:00) and other interdisciplinary meetings comprise peripheral clinical activities.

\section{Data collection and participants}

This study will use naturalistic observations, surveys, semistructured interviews and departmental metrics (eg, quality measures, patient severity scores and unit census) to collect a combination of quantitative and qualitative data. These data collection methods will be executed in parallel for a holistic and multidimensional understanding of the departmental context of information exchange. Inter-rater reliability (IRR) and recruitment activities will begin in February, and data collection is expected to be finalised by July. Data captured by each data collection method is summarised in table 1 and discussed below.

\section{Naturalistic observations}

Naturalistic observations of interdisciplinary rounds, within-discipline handover and peripheral clinical activities will take place to characterise the structured instances of information exchange in the PICU and CCCU. Two HF researchers will conduct observations on all days of the week, including weekends.

Interdisciplinary rounds are attended by a group that may include physicians, registered nurses $(\mathrm{RN})$, RTs, pharmacists, dietitians and surgeons. Families and patients are also encouraged to attend. As this study is descriptive rather than comparative in nature, statistical significance did not drive the determination of target participant sample size. Alternatively, we have defined an observation timeline with the intent of ensuring that the descriptive data generated are representative of the task and populations being studied. As attending physicians represent a key cohort of potential participants whose involvement in interdisciplinary rounds is instrumental to the planning and collaboration that occurs therein, the observation schedule was designed to maximise the 
Table 1 Data collection method and analysis overview

\section{Data collection method \\ Naturalistic DELTA Time-Motion Tool \\ observations}

Field Attendance form
notes

Action item table Quantitative. Descriptive analysis and summary statistics. High-level details of each patient's care plan as discussed at morning rounds and \% follow-up

Room map Qualitative. Descriptive analysis. Spatial orientation of rounding group, bedside

Free-text notes Qualitative. Thematic analysis. Details and context of observed events.

HCP interview

Family interview

HCP survey Closed-ended questions

Open-ended, free-text questions

Family survey Closed-ended questions
Open-ended, free-text
questions

Departmental metrics discussion regarding the care plan at afternoon rounds. clinicians, family and technology.

\section{Analysis and data captured}

Quantitative. Descriptive analysis, summary statistics. Duration of rounds and their specific elements (ie, case history, body system updates, education and so on), the role of participants, technology used and interruptions.

Quantitative. Descriptive analysis and summary statistics. Tracked attendance throughout rounds; number and type of clinicians present throughout rounds, at the bedside, and transiently; and prevalence of family attendance.

Qualitative. Thematic analysis. Perception of, expectations for and satisfaction with the current system.

Qualitative. Thematic analysis. Perception of, expectations for and satisfaction with the current system.

Quantitative. Descriptive analysis and summary statistics. Clinician demographics and categorical variables.

Qualitative. Thematic analysis. Perception of, expectations for and satisfaction with the current system.

Quantitative. Descriptive analysis and summary statistics. Categorical variables.

Qualitative. Thematic analysis. Perception of, expectations for and satisfaction with the current system.

Quantitative. Descriptive analysis, summary statistics. Contextualise observed and reported data.

HCP, healthcare provider.

number of attending physicians who may be observed in the study. At present, 16 attending physicians are divided between the two CCUs. Attending physicians will be assigned a random identification code at the start of the study and their attendance will be tracked during observations. Twenty-two fellows, approximately $300 \mathrm{RNs}$, 62 RTs, 7 pharmacists and 2 dietitians currently staff the CCU. Based on staffing estimates, advance scheduling, and discussion with clinical programme managers, the observation schedule will be arranged to try to maximise the number of unique staff encounters.

We will aim to observe interdisciplinary rounds led by each attending physician up to four times (two morning and two afternoon), totalling approximately 30 non-consecutive days. Based on the average duration of interdisciplinary rounds and unit census, we expect to observe over 100 interdisciplinary rounding hours and approximately 1000 patient encounters over the data collection period.

All within-discipline patient handovers and peripheral clinical activities will be observed until field note data have reached saturation; it is expected that saturation in the identification of issues or relevant behavioural variability will occur in approximately $5-7$ observations. ${ }^{33}$

Consent for all observational activities will follow an 'opt-out' model. This model was chosen after direct consultation with the SickKids Research Ethics Board. HCPs and family members will indicate if they wish to opt out as opposed to explicitly opting in. Easily accessible information about the nature of the study and the option and process to opt out will be provided to all HCPs and family members via posters in common areas. The CCU community will be additionally informed of the study by email and research team presence at intradisciplinary research rounds and education days. Multiple options for opting out will be available including email, phone call or in person to the researchers; SickKids staff will not be made aware of who has opted out. Observation scheduling will take place ahead of time in consultation with staff schedules; if possible, days when opted-out staff are working will not be included. The researchers will review the attending physician, medical trainee, RN and RT schedules prior to each observation to check if anyone who has opted out will be on shift, and if so, observation of the patient encounters involving that staff member will not take place. If incoming staff have opted out of observations, observation will stop for the remainder of the relevant encounters. Staff who have opted out will be asked to ensure that they display their badges prominently so that they are readily identifiable. Rounds on patients whose families have opted out will not be observed. The unit 
Table 2 DELTA application interface configuration

\begin{tabular}{|c|c|c|c|}
\hline Speaker & Content level 1 & Content level 2 & Content level 3 \\
\hline $\begin{array}{l}\text { Attending physician. } \\
\text { Fellow (presenting). } \\
\text { Fellow (other). } \\
\text { Bedside nurse. } \\
\text { Resident. } \\
\text { Pharmacist. } \\
\text { Clinical nurse specialist. } \\
\text { Charge nurse/Clinical support } \\
\text { nurse. } \\
\text { Respiratory therapist. } \\
\text { Nurse practitioner. } \\
\text { Family. } \\
\text { Dietitian. } \\
\text { Other. }\end{array}$ & $\begin{array}{l}\text { Patient encounter. } \\
\text { Patient intro/history. } \\
\text { Acute status update. } \\
\text { Care plan. } \\
\text { Prerounds. } \\
\text { Postrounds. }\end{array}$ & $\begin{array}{l}\text { Request info. } \\
\text { Correct info. } \\
\text { Teaching. } \\
\text { Interruption. } \\
\text { Logistics. } \\
\text { Discussion with family. } \\
\text { Non-patient-related } \\
\text { discussion. }\end{array}$ & $\begin{array}{l}\text { Interjection. } \\
\text { Central nervous system. } \\
\text { Cardiovascular system. } \\
\text { Access. } \\
\text { Respiratory. } \\
\text { Gastrointestinal. } \\
\text { Genitourinary. } \\
\text { Skin. } \\
\text { Labs. } \\
\text { Infectious disease. } \\
\text { Social. }\end{array}$ \\
\hline
\end{tabular}

census sheet will be consulted prior to rounds to determine a patient's presence and location within the CCU.

Time-motion data and qualitative field notes will be collected in parallel for morning and afternoon interdisciplinary rounds and nursing handovers. Field notes will be the primary data collected for all other activities. The research team will observe morning and afternoon interdisciplinary rounds in the same day to allow for analysis of care plan execution and continuity. The time-motion data will be used to collect time-stamped event data characterising the rounding process, while the field notes will serve to contextualise the time-motion data by capturing descriptive details surrounding the work system at the time of observation, including but not limited to room configuration and rounds attendance.

Together, these techniques will allow for the collection of a wide range of data during the observation of rounds, including: component tasks/processes of rounds, duration, spatial location, attendees, contributors, content discussed, manner of delivery, technology used, interpersonal interactions, family engagement and interruptions.

\section{Time-motion}

One of two observers from the research team will collect continuous time-motion data using an iPad running the application DELTA (Farzan Sasangohar, College Station, Texas). DELTA allows users to capture time-stamped event data across multiple dimensions, including workflow and communication patterns. ${ }^{34}$ Table 2 depicts a draft of the interface configuration of the tool. The researcher will create an event by selecting a variable from two or more of the columns. The data points that will be collected for each event are start time, end time, time elapsed and the variables selected from each column.

\section{Field notes}

A second observer from the research team will accompany the researcher collecting time-motion data to capture qualitative field notes. Data collection forms will guide the observation documentation: (1) an action item table will be used to collect high-level details of each patient's care plan as discussed at morning rounds and to track whether any follow-up discussion regarding the care plan occurs during afternoon rounds; (2) a room map will be used as needed to visually capture contextual details, such as the spatial position of people and technology in the room at the time of observation; (3) free-text notes will be collected and subsequently transcribed electronically; and (4) an attendance form will be used to track the attendance of all participants throughout the duration of rounds (see online supplementary files for data collection tools). Artefacts of clinical practice (eg, blank preprinted forms) may be collected, as well as photographs of the environment, technology or supplies.

\section{Inter-rater reliability}

Prior to the start of the formal data collection phase, the proposed DELTA interface configuration will be piloted in the SickKids CCU. Researchers will use the tool to observe both morning and afternoon rounds, and DELTA variables will be finalised by consensus of the researchers. Using the method of Ballermann et $a l^{35}$ IRR will be confirmed $(\kappa>0.85)$ between two researchers before either conducts their own time-motion observations.

To ensure the researchers develop an appropriate sensitivity to clinical details, a period of observer training will take place during which researchers will observe alongside an experienced SickKids clinician; all three observers will complete an action item table for each patient encountered on rounds until acceptable IRR scores are achieved (ie, $\kappa>0.7$ ).

The pilot period will serve to familiarise researchers with the data collection tools as well as the SickKids CCU.

\section{Surveys}

Surveys will be administered to HCPs to collect select demographic data and individual perceptions of and satisfaction with the current rounding processes in use at SickKids and to families to gather experiences of attending rounds from their perspective.

Surveys will be distributed to HCPs and parents or guardians of patients throughout the duration of the observation 
period to maximise response rate. They will be available to HCPs multimodally, through distribution by the research team at staff meetings, or distribution via a SickKids 'safety champion'. As the analysis to be applied to the survey data will be descriptive rather than statistically comparative in nature, no target sample size has been specified. Based on reported response rates for similar HCP survey studies published in the literature, a survey response rate between $55 \%$ and $95 \%$ is expected. ${ }^{2436}{ }^{37}$ Family surveys will be distributed by the research team at the bedside following rounds. Over 30 non-consecutive days, we expect to encounter approximately 180 different patients and their families. Similar studies involving family surveys have yielded response rates ranging from $29 \%$ to $100 \%$, depending on the method of survey distribution..$^{19248-40} \mathrm{In}$ a similar method to the proposed study, Levin $e t a l^{19}$ offered a survey to the present family member at the end of each rounding encounter, with a response rate of $87 \%$. Based on this rate, we expect to collect approximately 156 completed family surveys. A cover letter will be attached to each survey explaining the purpose of the study. Completion of the survey will be interpreted as consent for inclusion.

The HCP survey was derived from Holodinsky's National Survey of ICU Patient Care Rounds ${ }^{9}$ and Vats' Rounding Process Staff Satisfaction Survey, ${ }^{24}$ with additional questions to capture perspectives that complement objective time-motion and field note data. The questions focus on perceptions of rounds in general as they are currently implemented at SickKids (eg, What challenges or barriers do you come across to attending and participating in rounds? In your opinion, does the current rounding process promote patient-centredness? Should it?) The family survey was derived from Emerson's Family Discharge Survey ${ }^{38}$ and includes questions on families' perceived roles during rounds and level of satisfaction (eg, If you could change one thing about the current rounding process, what would it be?).

\section{Interviews}

Semistructured interviews will be conducted with the goal of clarifying observations and gathering detailed perceptions of rounds that are not feasible to collect through a survey. Five group interviews will be conducted, one with each of the following: (1) medical trainees, (2) RTs, (3) nurses, (4) pharmacists and (5) a combination of clinicians who do not frequently attend rounds (eg, dietitians, physiotherapists, social workers and patient service aides). Individual interviews will be held with CCU attending physicians and any non-CCU attending physicians who attend rounds (eg, surgeons and anaesthesiologists). Invitations to volunteer for interviews will be sent through the management of each profession. Interviews with patient families may be conducted immediately following each rounding encounter until qualitative saturation is reached (approximately 5-7 interviews). ${ }^{33}$ Written consent for interview participation will be collected via informed consent form by the research team prior to initiating each interview.
At least two researchers will be present during the interviews. HCP interviews will be audio-recorded and will be approximately 1 hour in length. Audio recordings will be transcribed by a member of the research team following the interview. On completion of rounds, researchers will also interview any families that wish to discuss the preceding round. Family interviews will last approximately $5 \mathrm{~min}$, and free-text notes will be collected during the discussion.

\section{Outcome measures}

The primary outcomes of this study will be:

1. Descriptive and quantitative characterisations of the current content, context and execution of structured information exchange activities in SickKids' CCU.

2. A descriptive list of codes (analytical framework) stratifying the following results according to SEIPS category and the relevant practice recommended by Lane et al, where applicable:

a. Effective practices and areas for improvement as observed and recorded in field notes.

b. Pervasive attitudes towards, perceptions and expectations of and overall satisfaction with structured information exchange activities based on stakeholder feedback from surveys and interviews.

\section{Data analysis}

Data analysis will be done following the data collection period until approximately September 2018.

\section{Quantitative analysis}

We propose the use of descriptive analysis and summary statistics to analyse closed-ended observational and survey data including but not limited to the duration of rounds and their specific elements (ie, case history, body system updates, discharge plan, education and so on), the number and role of participants, technology used and interruptions.

Time-motion data will be downloaded from the DELTA tool to Microsoft Excel, and attendance, room map and survey data will be entered manually following the completion of the study. Descriptive results, such as attendance at rounds, will be reported as percentages or means. Timemotion data from each unit, including average duration of rounds, average duration of rounding encounters, percentage of time spent on different elements of rounds and percentage of speaking time by rounding participants will be analysed in separate 2 (time of day; morning vs afternoon) $\times 2$ (time of week; weekend vs weekday) $\times 2$ (unit census; low vs high) $\times 2$ (patient acuity; low vs high) mixed factors analysis of variance with repeated measures on the first factor.

\section{Departmental metrics}

At the conclusion of the observation phase, the researchers will collect quality metrics that were tracked over observation period, as well as those collected over the preceding months and/or corresponding period of preceding years. The data will be used for reporting purposes and 
to compare and contextualise observation period averages. Metrics that have been identified as potentially tied to care team effectiveness, and therefore relevant to the study, include central line associated bloodstream infections, surgical site infections, length of stay, patient acuity and medication reconciliation on admission, transfer and discharge.

In addition, unit-specific census summary sheets will be collected by researchers from the nursing station at the time of each observation shift to capture the number of patients present in the unit throughout the study period. Descriptive statistics will be generated for the census volume data, which will serve to contextualise observation data (eg, if rounds take longer than usual, this may be explained by a high unit census).

\section{Multiframework thematic analysis}

A multiframework thematic analysis will be used to identify patterns in field observations and in the responses of clinicians and families with respect to their thoughts on and expectations of the rounding process.

A modified Framework Method ${ }^{41}$ will be used to analyse these data and seek to draw descriptive and/or explanatory conclusions clustered around themes. The general process we propose for implementing the Framework Method is summarised below and closely follows the steps outlined by Gale $e t$ al and Fan et al. ${ }^{41}{ }^{42}$ A description of data transformation for quantifying qualitative results is provided in steps 3-7.

1. At least two HF researchers will have been present at the time of all observations and interviews and will debrief to discuss their contextual or reflective impressions following each data collection session.

2. Qualitative data items (eg, hand-written field notes, free-text survey responses and recorded HCP interview responses) will be transcribed to digital text. Transcription will be completed by a member of the HF research team; one team member will be responsible for transcribing any one set of data items. All team members responsible for analysis (analysts) will review the complete transcripts to familiarise themselves with the collected data and contextual details of the session.

3. Analysts will independently assign codes to each transcribed item. These codes will be developed inductively and will be concise descriptions of the relevant item. Individual codes may be associated with multiple observed or reported items. Codes will then be grouped with other relevant codes by theme. To begin, themes will be predefined deductively and will include Lane et $a l$ 's Evidence-Informed Practices for Patient Care Rounds in the ICU. ${ }^{6}$ Where possible, codes will be labelled as either a facilitator or barrier to the implementation of the relevant best practice.

4. Following the review of a subset of transcripts, analysts will review their collective codes for overlap, come to consensus regarding wording moving forward and associate each code with the relevant SEIPS category. A priori themes will be assessed for their ability to cap- ture each of the codes identified. If these themes are not sufficient, they may be modified or added to with new inductive themes representative of the practices in place in SickKids' CCU. This set of codes and themes will comprise the analytical framework.

5. The analytical framework will be used and updated iteratively for subsequently coded transcripts. Analysts will retain the ability to add new codes if the present set fails to capture an observed or reported item. Analysts will convene to resolve discrepancies and agree on an updated analytical framework for subsequent analysis.

6. Once code development reaches saturation, an IRR test will be performed on a transcript, which each analyst has coded but not yet discussed. Analysts will repeat the process, coming to consensus and updating the analytical framework until an acceptable IRR (ie, $\mathrm{K}$ $>0.7$ ) is achieved and coding can justifiably proceed by any one analyst. Should subsequent transcripts necessitate adjustments to the analytical framework, the IRR process will be restarted. Previously coded transcripts will be updated retrospectively to ensure that the analytical framework is applied uniformly.

7. All coded items will be charted into a framework matrix for review by the research team. This matrix will organise codes by theme and sort items according to their code, method of collection and, where applicable, the individual patient encounter or attending physician interview. The matrix will concisely map items within and across data sets and facilitate analysis of their frequency and typology with helpful context. Figure 1 shows a sample framework matrix.

8. The charted data can then be analysed and interpreted to explore relationships, identify areas that are and are not functioning well within the department and potentially investigate causality and avenues for improvement.

\section{Interpretation of results}

Once the framework matrix is finalised, findings from each component of the study will be assessed for agreement (convergence), complementarity or apparent contradictions (dissonance). The subjective perceptions of different survey and interview participants will be compared with observational data to corroborate or contextualise observed phenomena and to highlight discrepancies between various stakeholder impressions and objective third-party observations. Examples of how quantitative and qualitative data may be integrated and interpreted to explore elements of information exchange are shown in Table 3. Based on this analysis, the research team will assess which of Lane $e t a l$ s practices have been implemented, with what observed and perceived success and as a result of which structural, procedural or contextual facilitators in the work system. Where established best practices are not applied, results will be examined to explore if and how similar results are achieved in this work system, or any barriers or direct opposition to the implementation of the recommendations. Novel, emerging 


\begin{tabular}{|c|c|c|c|c|c|c|c|c|c|}
\hline \multirow{2}{*}{\multicolumn{4}{|c|}{ Data Collection Method }} & \multicolumn{3}{|c|}{ Theme 1} & \multicolumn{2}{|c|}{ Theme ... } & \multirow{3}{*}{$\begin{array}{l}\text { E.g., Establish open and } \\
\text { collaborative discussion } \\
\text { environment }\end{array}$} \\
\hline & & & & Code A & $\mathrm{B}$ & C & $\mathrm{D}$ & $\mathrm{E}$ & \\
\hline \multirow{7}{*}{ 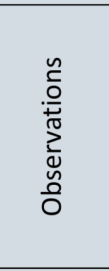 } & \multirow{3}{*}{ Rounds } & \multirow{3}{*}{ Patient } & 1 & & & & & & \\
\hline & & & 2 & & & & & & \multirow{5}{*}{$\begin{array}{l}\text { upportive communication } \\
\text { en members of different } \\
\text { ssions }\end{array}$} \\
\hline & & & $\ldots$ & & & & & & \\
\hline & \multirow{4}{*}{ Handover } & \multicolumn{2}{|l|}{ RN } & & & & & & \\
\hline & & \multicolumn{2}{|l|}{ RT } & & & & & & \\
\hline & & \multicolumn{2}{|c|}{ Charge RN } & & & & & & \\
\hline & & \multicolumn{2}{|c|}{ Charge RT } & & & & & & \multirow{9}{*}{$\begin{array}{l}\text { E.g., Attending physician thanks } \\
\text { bedside nurse and asks, "Do you } \\
\text { need anything else?" }\end{array}$} \\
\hline \multirow{9}{*}{ 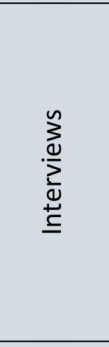 } & \multirow{3}{*}{\multicolumn{2}{|c|}{ Attending Physician }} & 1 & & & & & & \\
\hline & & & 2 & & & & & & \\
\hline & & & $\ldots$ & & & & & & \\
\hline & \multicolumn{3}{|l|}{ Fellow } & & & & & & \\
\hline & \multicolumn{3}{|l|}{$\mathrm{RN}$} & & & & & & \\
\hline & \multicolumn{3}{|l|}{ RT } & & & & & & \\
\hline & \multicolumn{3}{|l|}{ Pharmacist } & & & & & & \\
\hline & \multicolumn{3}{|l|}{ Other staff } & & & & & & \\
\hline & \multicolumn{3}{|l|}{ Family } & & & & & & \\
\hline \multirow{2}{*}{ Surveys } & \multicolumn{3}{|c|}{ Healthcare provider } & & & & & & \\
\hline & \multicolumn{3}{|l|}{ Family } & & & & & & \\
\hline
\end{tabular}

Figure 1 Framework matrix. Data items will be sorted by code (and theme), method of collection and individual patient encounter or attending physician interview where applicable. RN, registered nurse; RT, respiratory therapist.

effective practices and opportunities for improvement will be highlighted to inform future work.

\section{Patient and public involvement}

Information exchange in critical care was selected as a priority research area for its proven impact on patient outcomes and relation to the delivery of quality patient care. $^{22-25}$ Patients and public were not involved in the design of, recruitment to and conduct of the study; however, results from family surveys and interviews, along with representatives from the SickKids Family Advisory Network, will be incorporated into and involved in the development of safety-enhancing interventions following this study. Results of the study will be disseminated to study participants as per the dissemination plan below.

\section{ETHICAL CONSIDERATIONS}

We will collect a combination of electronic and paperbased data throughout the study. Physical documents including signed clinician consent forms, surveys and unit census sheets will be exclusively kept within the CCU at SickKids in a locked room in a locked drawer. Electronic data, including digital photographs taken on a dedicated camera, transcribed observation notes and survey/interview responses, and data collected using the time-motion software will be stored on password-protected and encrypted laptops. The collected data will be used to characterise systemic issues and will in no way be used to evaluate performance of the participants. Consent will be obtained for each aspect of data collection as discussed above. In data collected during observations, participants will only be referred to by their role (attending physician, fellow (presenting), fellow (other) and so on), except for attending physicians who will be assigned a random identification code. Observation notes will not contain any information related to the identity of patients or providers. Observations that could identify a particular person will not be recorded.

All surveys will be anonymous, confidential and will contain no participant identifiers. Although demographic information will be collected, it will be collected in categories that are sufficiently broad to ensure individuals are not easily identifiable (eg, specific age will not be collected but rather age category). Completed surveys will be kept in individually sealed envelopes to be opened and analysed only at the conclusion of the data collection phase. It will be ensured that the group interviews are well mediated, and all participants will be given the opportunity to contribute. No identifying data on patients or participants will be collected.

The researchers will be responsible for collecting data within the context of this project and are not expected to perform any clinical duties during the observation period. In the unlikely event that the researchers notice an error is about to be made, the researchers will intervene, but there is no expectation that they will look for and identify any and all errors that may occur during their observations.

\section{DISSEMINATION}

Findings from this study will be shared with the interdisciplinary CCU Steering Committee through a presentation given at a regularly scheduled quarterly meeting and/or a written report. Dissemination materials will be made available for circulation to the entire CCU staff. Smaller meetings with individual clinician groups will be scheduled by the HF research team to discuss findings and elicit feedback. In order to disseminate study results to participating families, a summary report will be available online; the URL will be distributed and available to families on information posters, survey 


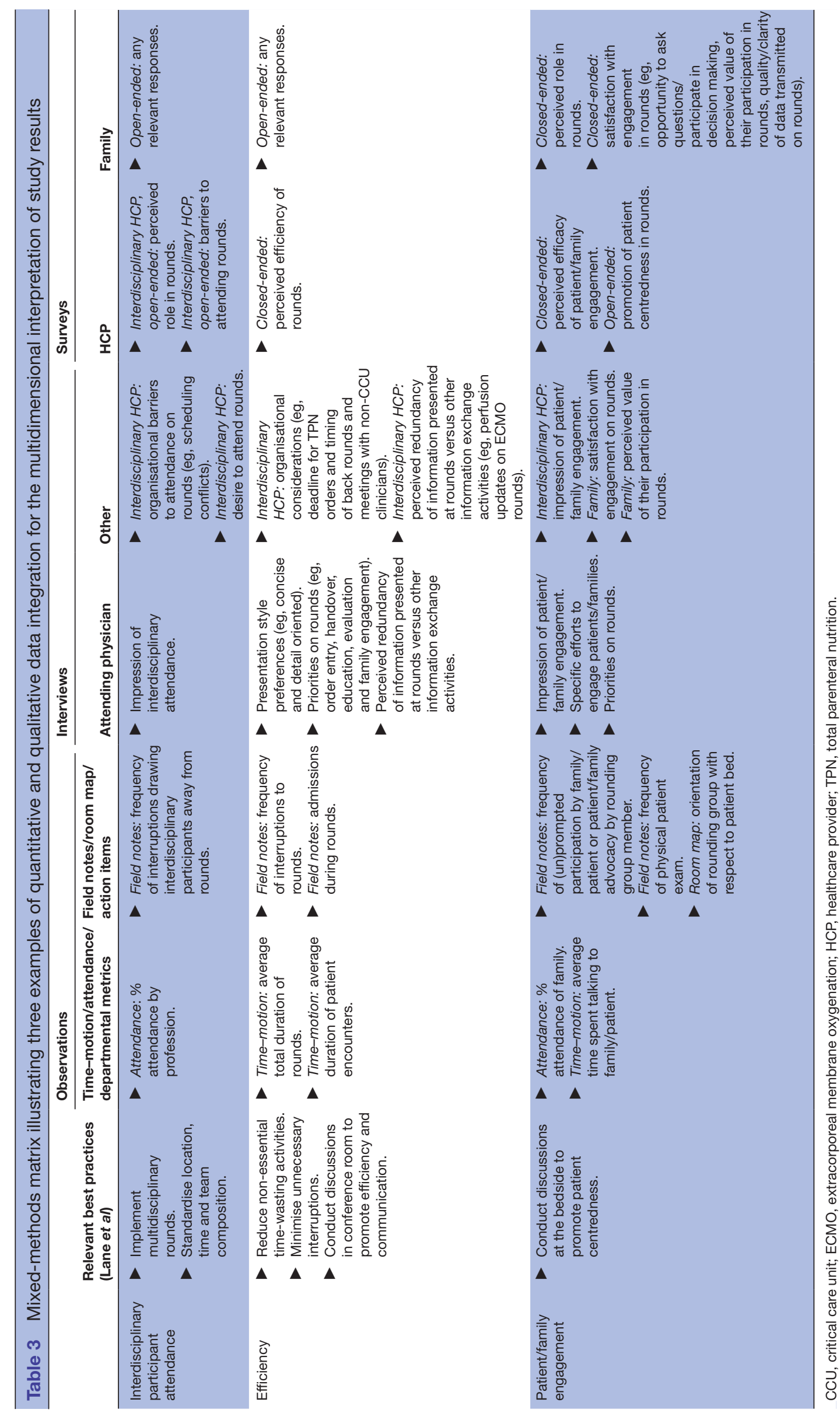

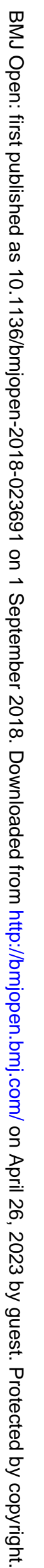


cover letters and interview informed consent forms. It is expected that the results of this study will be suitable for publication; a manuscript describing this study will be prepared for submission to a relevant peer-reviewed journal such as Critical Care Medicine. Finally, the findings will be used to redesign and improve aspects of rounds and information exchange, which in turn will be studied prospectively prior to official implementation following a similar methodology.

\section{LIMITATIONS}

As participation in this study is voluntary, a selection bias may exist as those who do not wish to participate may have similar characteristics and perceptions that cannot be captured. However, this bias should be limited based on the opt-out method of observation consent and high number of observation hours. Another potential limitation is that participants may alter their behaviour due to the presence of researchers; however, most observations will involve large groups in which the presence of two additional members will not stand out, and the researchers will not interact with participants during observations. Advance notice of the research via study recruitment materials may also affect participant behaviour.

Other potential limitations lie with the ability of the researchers. It is possible that some communication events may not be captured due to the complex and fast-paced CCU environment. Lastly, observations are limited to one department within one institution and may not be broadly applicable. However, the developed data collection tools and analytical framework may be adapted for use by other sites to embrace and understand the complexity of their own systems. The results of the present study may be used to benchmark against, and as information exchange represents a major component of any healthcare environment, it is expected that the results will be able to inform or inspire interventions at other departments or institutions.

\section{Author affiliations}

${ }^{1}$ HumanEra, Research and Innovation, North York General Hospital, Toronto, Ontario, Canada

${ }^{2}$ Institute of Biomaterials and Biomedical Engineering, University of Toronto, Toronto, Ontario, Canada

${ }^{3}$ Institute of Health Policy Management and Evaluation, University of Toronto, Toronto, Ontario, Canada

${ }^{4}$ Department of Critical Care Medicine, The Hospital for Sick Children, Toronto, Ontario, Canada

Acknowledgements The authors would like to thank project committee members Andrea McCormick, Winnie Seto and Brittany Lyn of The Hospital for Sick Children for their support in developing the study protocol and generating interest and encouraging participation from within their respective clinical units.

Contributors PL and PT are leading the study. JT, CW, LK, SP and PT drafted the study protocol; A-MG, RK, JH, CS and PS guided the study's clinical concept development, data collection material design and ethics board accountability. All authors critically reviewed and edited the final manuscript.

Funding This work was supported by the David and Stacey Cynamon Chair in Pediatric Critical Care at the Hospital for Sick Children.
Competing interests None declared.

Patient consent Not required.

Ethics approval This study has institutional research ethics board approval from The Hospital for Sick Children, Toronto, Ontario, Canada (SK REB: \#1000059173).

Provenance and peer review Not commissioned; externally peer reviewed.

Open access This is an open access article distributed in accordance with the Creative Commons Attribution Non Commercial (CC BY-NC 4.0) license, which permits others to distribute, remix, adapt, build upon this work non-commercially, and license their derivative works on different terms, provided the original work is properly cited, appropriate credit is given, any changes made indicated, and the use is non-commercial. See: http://creativecommons.org/licenses/by-nc/4.0/.

\section{REFERENCES}

1. Wilson RM, Runciman WB, Gibberd RW, et al. The quality in Australian health care study. Med J Aust 1995;163:458-71.

2. Leonard M, Graham S, Bonacum D. The human factor: the critical importance of effective teamwork and communication in providing safe care. Qual Saf Health Care 2004;13(Suppl 1):i85-90.

3. Coiera E, Tombs V. Communication behaviours in a hospital setting: an observational study. BMJ 1998:316:673-6.

4. Sutcliffe KM, Lewton E, Rosenthal MM. Communication failures: an insidious contributor to medical mishaps. Acad Med 2004;79:186-94.

5. Alameddine M, Dainty KN, Deber R, et al. The intensive care unit work environment: current challenges and recommendations for the future. J Crit Care 2009;24:243-8.

6. Lane D, Ferri M, Lemaire J, et al. A systematic review of evidenceinformed practices for patient care rounds in the ICU*. Crit Care Med 2013;41:2015-29.

7. Centofanti JE, Duan EH, Hoad NC, et al. Use of a daily goals checklist for morning ICU rounds: a mixed-methods study. Crit Care Med 2014;42:1797-803.

8. Donchin Y, Gopher D, Olin M, et al. A look into the nature and causes of human errors in the intensive care unit. Crit Care Med 1995;23:294-300.

9. Holodinsky JK, Hebert MA, Zygun DA, et al. A survey of rounding practices in Canadian adult intensive care units. PLoS One 2015; 10:e0145408.

10. Hefter $Y$, Madahar P, Eisen LA, et al. A time-motion study of ICU workflow and the impact of strain. Crit Care Med 2016;44:1482-9.

11. Tripathi S, Arteaga G, Rohlik G, et al. Implementation of patientcentered bedside rounds in the pediatric intensive care unit. J Nurs Care Qual 2015;30:160-6.

12. Mosby's Medical Dictionary. 8th edn. Milton, ON: Elsevier Health Sciences, 2008.

13. Paradis E, Leslie M, Gropper MA. Interprofessional rhetoric and operational realities: an ethnographic study of rounds in four intensive care units. Adv Health Sci Educ Theory Pract 2016;21:735-48.

14. Gonzalo JD, Heist BS, Duffy BL, et al. The value of bedside rounds: a multicenter qualitative study. Teach Learn Med 2013;25:326-33

15. Sandal S, lannuzzi MC, Knohl SJ. Can we make grand rounds "grand" again? J Grad Med Educ 2013;5:560-3.

16. O'Leary KJ, Buck R, Fligiel HM, et al. Structured interdisciplinary rounds in a medical teaching unit: improving patient safety. Arch Intern Med 2011;171:678-84.

17. Toeima E, Morris E. Improving patients' handover. Obstet Gynaecol Reprod Med 2013;23:221-3.

18. Friesdorf W, Konichezky S, Gross-Alltag F, et al. System ergonomic analysis of the morning ward round in an intensive care unit. $J$ Clin Monit 1994;10:201-9.

19. Levin $A B$, Fisher KR, Cato KD, et al. An evaluation of family-centered rounds in the PICU: room for improvement suggested by families and providers. Pediatr Crit Care Med 2015;16:801-7.

20. Vats A, Goin KH, Fortenberry JD. Lean analysis of a pediatric intensive care unit physician group rounding process to identify inefficiencies and opportunities for improvement. Pediatr Crit Care Med 2011:12:415-21.

21. Gangadharan S, Belpanno B, Silver P. An analysis of the daily workrounding process in a pediatric intensive care unit. $J$ Healthc Qual 2017;39:122-7.

22. Rathert C, May DR. Health care work environments, employee satisfaction, and patient safety: Care provider perspectives. Health Care Manage Rev 2007;32:2-11. 
23. Mosadeghrad AM. Factors influencing healthcare service quality. Int $J$ Health Policy Manag 2014;3:77-89.

24. Vats A, Goin KH, Villarreal MC, et al. The impact of a lean rounding process in a pediatric intensive care unit. Crit Care Med 2012;40:608-17

25. Ham PB, Anderton T, Gallaher R, et al. Development of electronic medical record-based "Rounds Report" results in improved resident efficiency, more time for direct patient care and education, and less resident duty hour violations. Am Surg 2016;82:853-9.

26. Arora V, Johnson J, Lovinger D, et al. Communication failures in patient sign-out and suggestions for improvement: a critical incident analysis. Qual Saf Health Care 2005;14:401-7.

27. Borowitz SM, Waggoner-Fountain LA, Bass EJ, et al. Adequacy of information transferred at resident sign-out (in-hospital handover of care): a prospective survey. Qual Saf Health Care 2008;17:6-10.

28. Collins S, Hurley AC, Chang FY, et al. Content and functional specifications for a standards-based multidisciplinary rounding tool to maintain continuity across acute and critical care. J Am Med Inform Assoc 2014;21:438-47.

29. Collins SA, Bakken S, Vawdrey DK, et al. Model development for EHR interdisciplinary information exchange of ICU common goals. Int J Med Inform 2011;80:e141-e149.

30. Easty T, Griffin M, Cassano-Piche A, et al, 2011. Module 2: human factors design: applications to healthcare. The patient saftey education program Canada. http://www.patientsafetyinstitute.ca/ en/education/PatientSafetyEducationProgram/PatientSafetyEdu cationCurriculum/pages/module-2-human-factors-design.aspx (accessed Mar 2018).

31. Holden RJ, Carayon P, Gurses AP, et al. SEIPS 2.0: a human factors framework for studying and improving the work of healthcare professionals and patients. Ergonomics 2013;56:1669-86.

32. Laussen P, Hubbert J. Critical care medicine. SickKids. http://www. sickkids.ca/Critical-Care/welcome/index.html (Date accessed: 2018/03).
33. Nielsen J, Landauer TK. A mathematical model of the finding of usability problems. Proceedings of ACM INTERCHI'93 Conference. Amsterdam, 1993

34. Sasangohar F, Donmez B, Easty AC, et al. Mitigating nonurgent interruptions during high-severity intensive care unit tasks using a task-severity awareness tool: A quasi-controlled observational study. J Crit Care 2015;30:1150.e1-6.

35. Ballermann MA, Shaw NT, Mayes DC, et al. Validation of the Work Observation Method By Activity Timing (WOMBAT) method of conducting time-motion observations in critical care settings: an observational study. BMC Med Inform Decis Mak 2011;11:32.

36. Genet IC, Firestone KS, Volsko TA. Neonatal respiratory therapist-led rounds can improve staff satisfaction and timeliness of respiratory interventions. Respir Care 2015;60:321-7.

37. Rehder KJ, Uhl TL, Meliones JN, et al. Targeted interventions improve shared agreement of daily goals in the pediatric intensive care unit. Pediatr Crit Care Med 2012;13:6-10.

38. Emerson BL, Chmura KB, Walker D. Hourly rounding in the pediatric emergency department: patient and family safety and satisfaction rounds. J Emerg Med 2014;47:99-104.

39. Schiller WR, Anderson BF. Family as a member of the trauma rounds: a strategy for maximized communication. J Trauma Nurs 2003;10:93-101.

40. Voos KC, Ross G, Ward MJ, et al. Effects of implementing familycentered rounds (FCRs) in a neonatal intensive care unit (NICU). $J$ Matern Fetal Neonatal Med 2011;24:1403-6.

41. Gale NK, Heath G, Cameron E, et al. Using the framework method for the analysis of qualitative data in multi-disciplinary health research. BMC Med Res Methodol 2013;13:117.

42. Fan M, Petrosoniak A, Pinkney S, et al. Study protocol for a framework analysis using video review to identify latent safety threats: trauma resuscitation using in situ simulation team training (TRUST). BMJ Open 2016;6:e013683. 